\title{
Nanoindentation Response of Polycrystalline Graphene by Atomistic Simulations
}

\author{
Ying $L^{1, a}$ and Jin QIAN ${ }^{1,2, b, *}$ \\ ${ }^{1}$ Department of Engineering Mechanics, Zhejiang University, Hangzhou, Zhejiang 310027, China \\ ${ }^{2}$ Key Laboratory of Soft Machines and Smart Devices of Zhejiang Province, Zhejiang University, \\ Hangzhou, Zhejiang 310027, China \\ ayinglu@zju.edu.cn, bjqian@zju.edu.cn \\ *Corresponding author
}

Keywords: Polycrystalline graphene, Nanoindentation, Mechanical properties, Molecular dynamics

\begin{abstract}
Nanoindentation has been widely used to determine the mechanical properties of pristine and polycrystalline graphene in experiments. To investigate the effects of grain size on the mechanical properties of polycrystalline graphene, we use molecular dynamics simulations to study the nanoindentation response of suspended polycrystalline graphene layer. The force-displacement behavior from the simulations enables the interpretation of Young's modulus and breaking strength of the 2D material, which are exhibited to strongly depend on the grain size.
\end{abstract}

\section{Introduction}

Pristine graphene with perfectly hexagonal carbon rings exhibits extraordinary mechanical properties. However, large-scale monolayers of graphene grown by chemical vapor deposition (CVD) usually contain topological defects and grain boundaries, which might strongly influence the apparent properties of the 2D material. There have been increasing experimental and simulation studies on the stiffness and strength of polycrystalline graphene. For example, atomic force microscopy (AFM) has been employed to measure the mechanical properties of monolayer-graphene in a nanoindentation setup, leading to the interpretation of 2D elastic modulus and breaking strength, respectively [1,2]. Both pristine and polycrystalline graphene (with varying grain sizes) have been measured by nanoindentation in AFM, and it was shown that the stiffness and failure load of poly-graphene are comparable with those of pristine graphene, despite the existence of grain boundaries [2]. In contrast, some other studies showed that grain boundaries severely reduce the elastic modulus and fracture strength of graphene [3,4]. For nanoindentation technique, the experimental measuremens and simulation predictions on the modulus and strength of polycrystalline graphene were found to be scattered widely [5-8], which were also different from those by uniaxial tension [9,10]. This work aims to explore the role of grain size in influencing the mechanical properities of polycrystalline graphene. Molecular dynamics (MD) simulations are employed to perform the nanoindentation test in mimicking the experimental measurements in AFM.

\section{Indentation of Polycrystalline Graphene: Atomistic Simulations}

In experiments, polycrystalline graphene is usually generated by chemical vapor deposition, in which the growth of graphene monolayers starts from multiple nucleation sites simultaneously. As such, the orientations of individual grains rely on the local nucleation sites and are randomly distributed [11]. To account for such growth, we perform computational procedure to generate a certain number $N$ of randomly placed seeds within a square region, and use Voronoi tessellation method to divide the area into $N$ polygons. Each polygon is filled by a pristine graphene fragment with a random orientation angle $\theta_{\mathrm{i}}$, which is represented by the angle between the armchair direction and the horizontal direction. Owing to the hexagonal honeycomb structure of graphene, the angle $\theta_{\mathrm{i}}$ falls into the range between $-\pi / 6$ and $\pi / 6$. The atoms near grain boundary junctions that are closer than $1.4 \AA$ to any 
neighboring grains are removed. This treatment is based on the observation that the system with grain boundary atoms closer than this value is unstable. In this way, the coordinates of all carbon atoms in polycrystalline graphene are generated within a simulation cell (selected as $20 \mathrm{~nm}$ by $20 \mathrm{~nm}$ ). Fig. 1(a) shows the topological structure of a polycrystalline graphene sample with the mean grain size of $2 \mathrm{~nm}$, which contains 127 grains in total. A circular region with radius $R$ is selected from the sample for nanoindentation simulations, in which the atoms along the periphery are fixed as boundary condition (Fig. 1(b)). We confirm that the distribution of misorientation angle from this sample preparation resembles a random distribution, meaning that there is no preferred orientation for the grains (Fig. 1(c)). The distribution of grain size is Gaussian with the average value of $2 \mathrm{~nm}$, as shown in Fig. 1(d). The polycrystalline graphene samples with varying grain sizes can be readily produced by changing the number $N$ of nucleation sites within the given area.

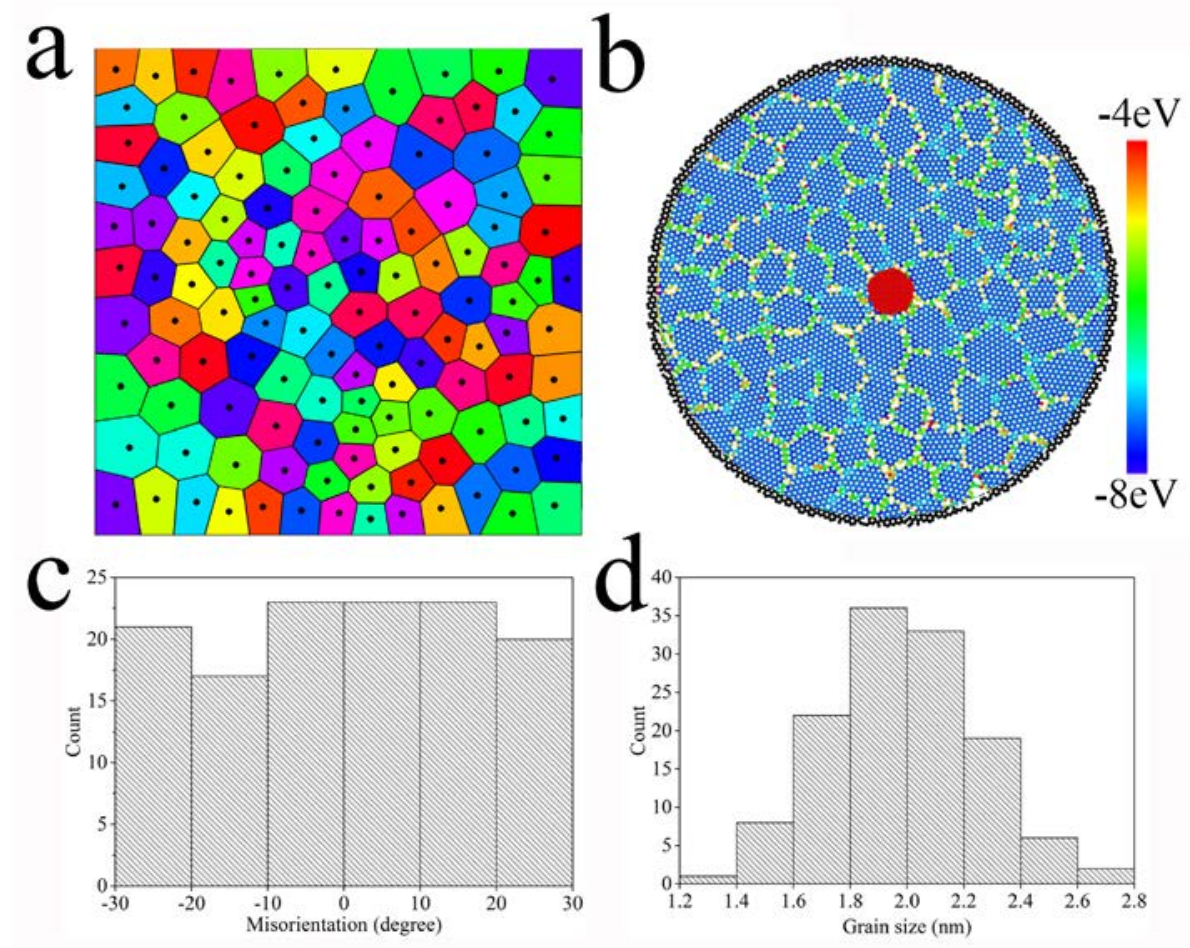

Fig. 1 (a) The topological structure of polycrystalline graphene with the average grain size of $2 \mathrm{~nm}$. The simulation cell ( $20 \mathrm{~nm}$ by $20 \mathrm{~nm}$ ) contains 127 grains that are randomly oriented. Colors in the figure are used to distinguish neighboring grains. (b) Nanoindentation simulation of a suspended polycrystalline graphene layer. The atoms along the periphery (in black) are fixed during the simulation, and the color bar represents the energy of the atoms. The distributions of (c) the misorientation angle and (d) the grain size of the sample in (a).

Next, we use molecular dynamics simulations to study the nanoindentation behavior of such polycrystalline graphene. A circular membrane of polycrystalline graphene (Radius: $R=10 \mathrm{~nm}$ ) is perpendicularly indented by a hemispherical diamond tip (Radius: $r=1 \mathrm{~nm}$ ) at the membrane center, as shown in Fig. 1(b). Our nanoindentation simulations were performed at constant atom number (N), volume (V) and temperature (T) (i.e., NVT ensemble) by using the large-scale atomic/molecular massively parallel simulator (LAMMPS). The system temperature was maintained at $1 \mathrm{~K}$ by employing Nos'e-Hoover thermostat, and the initial velocities of atoms in each direction were extracted from Gaussian distribution for the given temperature. The adaptive intermolecular reactive empirical bond order (AIREBO) potential [12] was used for carbon-carbon interaction in the simulations, with a switch function parameter $2.0 \AA$ for the breakage of C-C bonds [13]. For the atomic-scale interaction between the atoms from the indenter tip and graphene layer, respectively, Lennard-Jones potential was used to describe the van der Walls type interaction, namely, 


$$
U(r)=4 \varepsilon\left\{(\sigma / r)^{12}-(\sigma / r)^{6}\right\}
$$

where $\varepsilon$ is the depth of the potential well and $\sigma$ is the finite distance at which the inter-atom potential is zero, and $r$ is the distance between the atoms. $\varepsilon=2.85 \mathrm{meV}$ and $\sigma=0.34 \mathrm{~nm}$ were selected in our simulations [14], and $r_{c}=3.5 \sigma$ was used for the cutoff distance for the Lennard-Jones interaction to vanish. In our simulations, the indenter tip was pushed against the graphene layer at a velocity of $10 \mathrm{~nm} / \mathrm{ns}$ after the system was fully relaxed. A constant time step of $0.5 \mathrm{fs}$ was used in all the simulations unless stated otherwise.

\section{Results and Discussion}

Nanoindentation is an indirect measurement on the mechanical properties of 2D materials, and a theoretical analysis is needed for the extraction of material stiffness and strength. In the present case, the lateral size of the poly-graphene membrane under investigation $(20 \mathrm{~nm})$ is much larger than the indentation depth (usually $<3 \mathrm{~nm}$ ), and the membrane thickness of monolayer graphene $(0.34 \mathrm{~nm})$ is much smaller than the indentation depth. Following the nonlinear elasticity theory of a clamped circular flake under perpendicular indentation, the quantitative relation between the applied force $(F)$ and indentation depth $(\delta)$ is $[1,15,16]$

$$
F=\sigma_{0}^{2 \mathrm{D}}(\pi R)(\delta / R)+E^{2 \mathrm{D}}\left(G^{3} R\right)(\delta / R)^{3},
$$

where $\sigma_{0}^{2 \mathrm{D}}$ is the pre-tension in the graphene layer, $E^{2 \mathrm{D}}$ is the $2 \mathrm{D}$ Young's modulus, and $G=\left(1.05-0.15 v-0.16 v^{2}\right)^{-1}$ is a numerical factor related to Poisson's ratio $v . v=0.165$ for graphite in the basal plane was taken in our analysis.

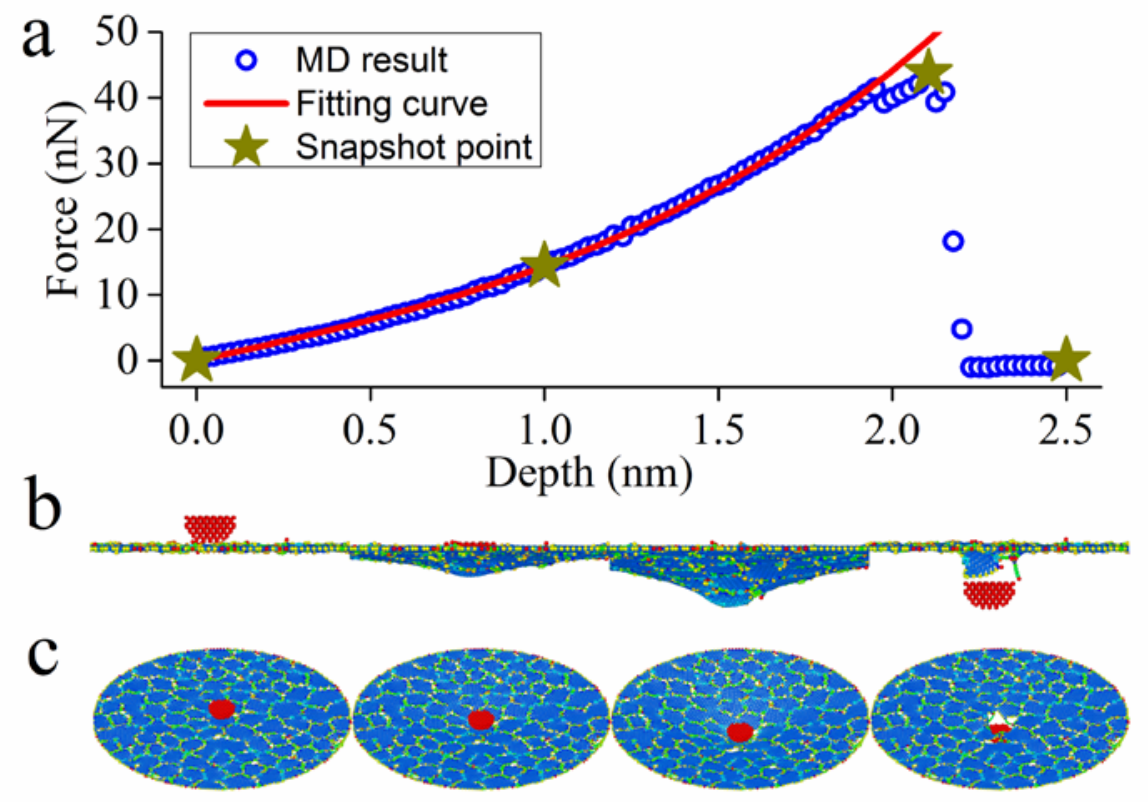

Fig. 2 (a) The curve of force versus depth during the nanoindentation process. The circles (blue) are simulation results and the solid line (red) is the theoretical interpretation. (b) The side-view and (c) top-view snapshots of the poly-graphene and indenter when the indentation depth are 0,1, 2.1 and 2.5 $\mathrm{nm}$, respectively, which are indicated by pentagrams in (a).

Fig. 2(a) shows one representative behavior of force versus indentation depth, where the blue circles are simulation results. The force increases with the indentation depth until a maximum force (denoted as $F_{\max }$ ) is reached, corresponding to the critical failure of graphene layer. The maximum 
stress (i.e., the breaking strength) within the clamped circular graphene membrane under a hemispherical indenter tip can be calculated as [1]

$$
\sigma_{\max }^{2 \mathrm{D}}=\sqrt{F_{\max } E^{2 \mathrm{D}} /(4 \pi r)}
$$

where $E^{2 \mathrm{D}}$ is determined by matching the theoretical model of Eq. 2 to the force-depth response of MD simulations before the occurance of failure. In particular, the 2D Young's modulus of the poly-graphene sample with grain size of $2 \mathrm{~nm}$ is $178 \mathrm{~N} / \mathrm{m}$, which can be converted to 524 GPa by taking the membrane thickness of $0.34 \mathrm{~nm}$. The $2 \mathrm{D}$ breaking strength $\left(\sigma_{\max }^{2 \mathrm{D}}\right)$ of the poly-graphene sample is calculated to be $24.7 \mathrm{~N} / \mathrm{m}$ from Eq. 3, corresponding to $72.6 \mathrm{GPa}$. For comparison, we also performed nanoindentation simulations for pristine graphene, yielding Young's modulus and breaking strength of $1.05 \mathrm{TPa}$ and $126 \mathrm{GPa}$, respectively. These simulation results for pristine graphene are consistent to the experimental measurements [1]. Several snapshots of the nanoindentation simulation process are shown in Figs. 2(b) and 2(c).

For the same average grain size of $2 \mathrm{~nm}$, we conducted simulations on ten different samples of polycrystalline graphene with random topology in order to obtain the statistical information on the mechanical properties, and Young's modulus and breaking strength from the collection are calculated to be $E=656 \pm 103 \mathrm{GPa}$ and $\sigma_{\max }=71 \pm 6 \mathrm{GPa}$ (mean values with standard deviations). When the average grain size is changed to $5 \mathrm{~nm}$, ten-sample simulations yield $E=915 \pm 117 \mathrm{GPa}$ and $\sigma_{\max }=94 \pm 14.7 \mathrm{GPa}$. Figs. 3(a) and 3(b) show the box-plots of these results for polycrystalline graphene with two grain sizes, both being significantly lower than the corresponding results for pristine graphene.
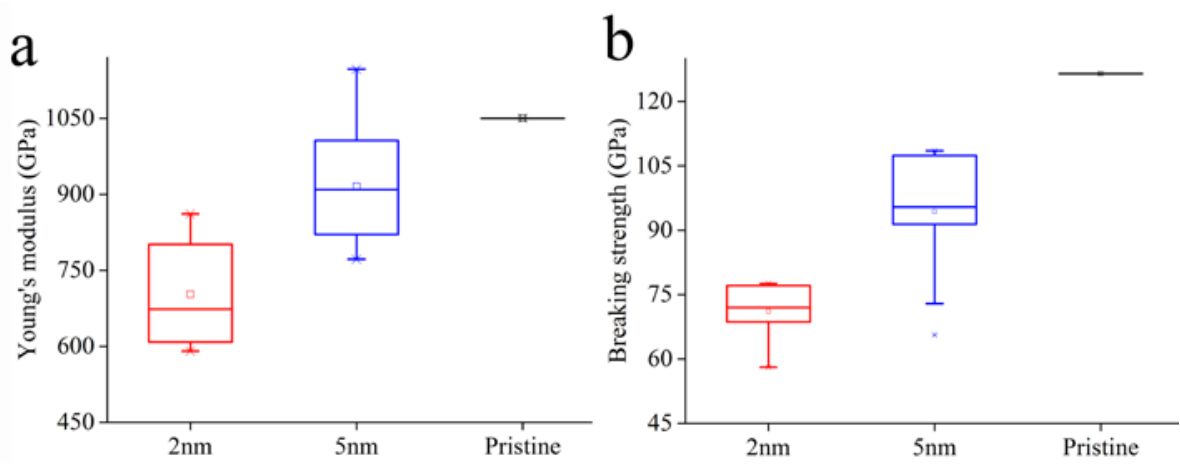

Fig. 3 Statistical summary of the simulation results on the mechanical properties of poly-graphene. The box-plots of (a) Young's modulus and (b) breaking strength for polycrystalline graphene with two grain sizes of $2 \mathrm{~nm}$ and $5 \mathrm{~nm}$ are compared with those of pristine graphene. Each plot includes the sample minimum, lower quartile, median, upper quartile and sample maximum.

\section{Summary}

In this study, we have performed atomistic simulations of nanoindentation to explore the grain size-dependent mechanical properties of polycrystalline graphene. The main finding is that the interpretations of Young's modulus and breaking strength of poly-graphene from nanoindentation are strongly influenced by the grain size. The presence of grain boundaries significantly weakens the 2D material, which should arise from the combination of grain boundary juntions, holes and other defects in the structure. This is evident by the fact that the failure of polycrystalline graphene always occurs at grain boundary juntions in our simulations. Accumulating results elucidating the nanoscopic mechanisms leading to the determinant role of grain size in affecting the poly-graphene properties are underway. 


\section{Acknowledgement}

This work was supported by the National Natural Science Foundation of China (No. 11321202) and the Zhejiang Provincial Natural Science Foundation of China (No. LR16A020001).

\section{References}

[1] C. Lee, X.D. Wei, J.W. Kysar, J. Hone, Measurement of the elastic properties and intrinsic strength of monolayer graphene, Science 321 (2008) 385-388.

[2] G.H. Lee, R.C. Cooper, S.J. An, S. Lee, A. van der Zande, N. Petrone, A.G. Hammerherg, C. Lee, B. Crawford, W. Oliver, J.W. Kysar, J. Hone, High-strength chemical-vapor deposited graphene and grain boundaries, Science 340 (2013) 1073-1076.

[3] P.Y. Huang, C.S. Ruiz-Vargas, A.M. van der Zande, W.S. Whitney, M.P. Levendorf, J.W. Kevek, S. Garg, J.S. Alden, C.J. Hustedt, Y. Zhu, J. Park, P.L. McEuen, D.A. Muller, Grains and grain boundaries in single-layer graphene atomic patchwork quilts, Nature 469 (2011) 389-392.

[4] C.S. Ruiz-Vargas, H.L.L. Zhuang, P.Y. Huang, A.M. van der Zande, S. Garg, P.L. McEuen, D.A. Muller, R.G. Hennig, J. Park, Softened elastic response and unzipping in chemical vapor deposition graphene membranes, Nano Lett. 11 (2011) 2259-2263.

[5] M. Neek-Amal, F.M. Peeters, Nanoindentation of a circular sheet of bilayer graphene, Phys. Rev. B 81 (2010) 235421.

[6] X.J. Tan, J. Wu, K.W. Zhang, X.Y. Peng, L.Z. Sun, J.X. Zhong, Nanoindentation models and Young's modulus of monolayer graphene: a molecular dynamics study, Appl. Phys. Lett. 102 (2013) 071908.

[7] R. Perriot, X. Gu, Oleynik, II, Computational nanomechanics of graphene membranes, in: N. Tamura, A. Minor, C. Murray, L. Friedman (Eds.), Probing Mechanics at Nanoscale Dimensions, Materials Research Society, 2009, pp. 45-50.

[8] Z.D. Sha, Q. Wan, Q.X. Pei, S.S. Quek, Z.S. Liu, Y.W. Zhang, V.B. Shenoy, On the failure load and mechanism of polycrystalline graphene by nanoindentation, Sci. Rep. 4 (2014) 7437.

[9] J. Kotakoski, J.C. Meyer, Mechanical properties of polycrystalline graphene based on a realistic atomistic model, Phys. Rev. B 85 (2012) 195447.

[10] A.J. Cao, J.M. Qu, Atomistic simulation study of brittle failure in nanocrystalline graphene under uniaxial tension, Appl. Phys. Lett. 102 (2013) 071902.

[11] Q.K. Yu, L.A. Jauregui, W. Wu, R. Colby, J.F. Tian, Z.H. Su, H.L. Cao, Z.H. Liu, D. Pandey, D.G. Wei, T.F. Chung, P. Peng, N.P. Guisinger, E.A. Stach, J.M. Bao, S.S. Pei, Y.P. Chen, Control and characterization of individual grains and grain boundaries in graphene grown by chemical vapour deposition, Nature Mater. 10 (2011) 443-449.

[12] S.J. Stuart, A.B. Tutein, J.A. Harrison, A reactive potential for hydrocarbons with intermolecular interactions, J. Chem. Phys. 112 (2000) 6472-6486.

[13] Z.P. Xu, Graphene nano-ribbons under tension, J. Comp. Theo. Nanosci. 6 (2009) 625-628.

[14] R.S. Ruoff, A.P. Hickman, van der Waals binding of fullerenes to a graphite plane, J. Phys. Chem. 97 (1993) 2494-2496.

[15] U. Komaragiri, M.R. Begley, The mechanical response of freestanding circular elastic films under point and pressure loads, J. App. Mech. 72 (2005) 203-212. 
[16] K.T. Wan, S. Guo, D.A. Dillard, A theoretical and numerical study of a thin clamped circular film under an external load in the presence of a tensile residual stress, Thin Solid Films 425 (2003) 150-162. 